\title{
ESTUDO SOBRE UM MÉTODO DE IRRIGAÇĀO INTESTINAL EM COLOSTOMIZADOS
}

Vera Lucia de Gouveia Santos*

\section{NOTA PREVIA}

SANTOS, V. L. C. de G. Estudo sobre um método de irrigação intestinal em colostomizados. Nota prévia. Rev. Esc. Enf. USP, São Paulo.

No Brasil, a problemática da readaptação do paciente colostomizado à sociedade vem constituindo motivo de preocupação para os profissionais da área de saúde.

O cuidado da pele e equipamento, que inclui como aspecto fundamental o controle das eliminações intestinais, ainda é embasado em tentativa e erro, retardando o auto-cuidado e conseqüente ajustamento gradativo do paciente às atividade sociais e hábitos diários de vida.

Para o colostomizado, a ausência de vazamentos e odores e portanto,, o controle das exoneraçōes pelo estoma, é de significadc vital. Esse controle é um dos fatores fundamentais capaz de transportá-lo da condi. ção de paciente para a de indivíduo normal.

O controle das eliminações, pode ser feito usando equipamento específico e/ou métodos de controle do próprio hábito intestinal. Quanto a este último, dois métodos são mais conhecidos: o controle natural e a irrigação intestinal. A irrigação intestinal, tem sido a alternativa de eleição em vários locais como Estados Unidos e Europa, em função de seus ótimos resultados $1,3,4,5,6,7,8,9$.

Conceitualmente, a irrigação é um enema cujo fluído enviado para o intestino grosso, promove sua irrigação, estimula sua peristalse e conseqüentemente esvazia seu conteúdo fecal *. A irrigação, para esse caso, é feita através do estoma.

Autores como GRIER *, SEARGENT ', MAZIER et alii ', AMDRUP \& CHRISTENSEN ', TERRANOVA ${ }^{8}$, GABRIELLI et alii ", além de WILLIAMS \& JOHNSTON ${ }^{9}$, DORAN \& HARDCASTLE ${ }^{2}$, entre outros, elaboraram estudos comparativos entre os métodos natural e de irrigação. O tratamento por irrigação, com pequenas restrições, foi sempre superior e mais eficiente.

* Auxiliar de Ensino do Departamento de Enfermagem Médico-Cirúrgica da Escola de Enfermagem da USP - disciplina Enfermagem Médico-Cirúrgica. 
Cumpre lembrar que para utilizar a irrigação é necessário analisar previamente aspectos tais como idade, hábitos intestinais anteriores à cirurgia, doença de base e caracteristicas estomais e periestomais $3,5,6,8,9$.

Considerando-se a necessidade da melhoria na qualidade de vida pós-operatória, além da importância do controle do hábito intestinal para o ostomizado e da irrigação como método mais propício para isso, propomo-nos a efetuar este estudo por meio de um método adaptado de irrigação. Este tem por finalidade diminuir o custo do equipamento utilizado para a irrigação sem alterar o efeito terapêutico desejado. Conseqüentemente seu uso poderia ser difundido nos ambientes hospitalar, ambulatorial e domiciliar para a assistência aos colostomizados.

O estudo encontra-se em fase de coleta de dados e está sendo efetuado no Serviço Ambulatorial de Colo-Proctologia de um Hospital Geral.

SANTUS, V. L. C. de G. Study of intestinal irrigation of colostomy patients: previous note. Rev. Esc. Enf. USP, Sã̃o Paulo.

The author relates the problems of colostomy patient during the rehabilitation, intestinal control, adequate equipment and skin care. She refers to the control of intestinal habit with colostomy irrigation and relates comparative studies that demonstrated the efficiency and superiority of this method in relation to natural evacuation. She proposes an experimental sistematized study with adaptable and cheap equipment which will not interfere in the therapeutic effect of colostomy and will be used in hospital, ambulatory and at home.

The data of this study is being collected in the Proctology Ambulatorial Service of a General Hospital.

\section{REFERENCIAS BIBLIOGRĀFICAS}

1. AMDRUP, E. \& CHRISTENSEN, P. Colostomy care: natural evacuation or irrigation? Amer.' J. Dig. Dis., New York, 12(7):747-8, 1967.

2. DORAN, J. \& HARDCASTLE, D. J. A controlled trail of colostomy management by natural evacuation, irrigation and foam enema. Brit. J. Surg., Bristol, 68(10):731-3, 1981.

3. GABRIELLI, F. et alil. Risultati dell'irrigazione periodica nella riabilitazione del colostomizzato. Minerva Chir., Torino, $35(21): 1649-64,1980$.

4. GRIER, N. W. R. et alil. An evaluation of colonic stoma management without irrigations. Surg. Gynec. Obstet., Chicago, 118(6):1234-42, 1964.

5. MAC LEOD, J. H. Colostomy irrigation: o transatlantic controversy. Dis. Colon. Rect., Philadelphia, 15(5):357-60, 1972.

6. MAZIER, W. P. et alli. Effective colostomy irrigation. Surg. Gynec. Obstet., Chicago, 142:905-9, June 1976 .

7. SEARGENT, P. W. Colostomy management by irrigation technique: review of 165 cases. Brit. Med. J., London, 2(5504):25-6, July 1966.

8. TERRANOVA, $O$. et alil. Irrigation vs natural evacuation of left colostomy: a comparative study of 340 patients. Dis. Colon Rect., Philadelphia. 22(1):31-4, 1979.

9. WILLIAMS, N. S. \& JOHNSTON, D. Prospective controlled trial comparing colostomy irrigation with «spontaneous action» method. Brit. Med. J., London, 281(6233):107-9, 1980. 